\title{
Layer-Dependent Band Structure of Ternary Metal Chalcogenides: Thickness-Controlled Hexagonal FeIn ${ }_{2} \mathrm{~S}_{4}$
}

Hyunjung Kim ${ }^{1,2}$, Xinghui Liü ${ }^{1,3}$, Meeree Kim ${ }^{1,3}$, Yunhee Cho ${ }^{1,3}$, JinJu Lee ${ }^{1,3}$, Thi Anh Le ${ }^{1,3}$, Ngoc Quang $\operatorname{Tran}^{1}$, Amol Jadhav ${ }^{1}$, and Hyoyoung Lee ${ }^{1,2,3,4^{*}}$

${ }^{1}$ Center for Integrated Nanostructure Physics (CINAP), Institute for Basic Science (IBS), 2066 Seoburo, Jangan-gu, Suwon 16419, Republic of Korea, ${ }^{2}$ Sungkyunkwan University Advanced Institute of Nano Technology (SAINT), Sungkyunkwan University (SKKU), 2066 Seoburo, Jangan-gu, Suwon 16419, Republic of Korea, ${ }^{3}$ Department of Chemistry, Sungkyunkwan University (SKKU), 2066 Seoburo, Jangan-gu ,Suwon 16419, Republic of Korea, ${ }^{4}$ Department of Biophysics, Sungkyunkwan University (SKKU), 2066 Seoburo, Jangan-gu, Suwon 16419, Republic of Korea 


\section{Step 1)}

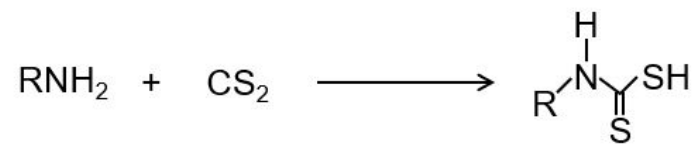

\section{Step 2)}

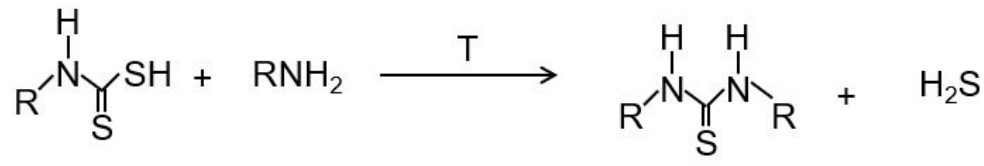

Scheme S2. The mechanisms of chalcogen dissociation by $\mathrm{CS}_{2}$ injection in the synthesis of the nanocrystal.
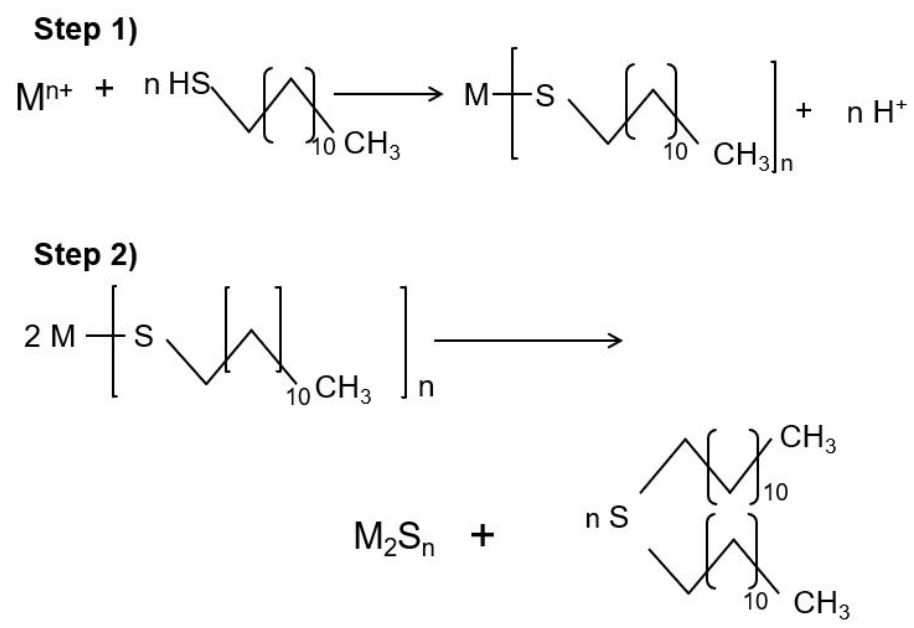

Scheme S1. The mechanisms of chalcogen dissociation by DDT injection in the synthesis of nanocrystals. 


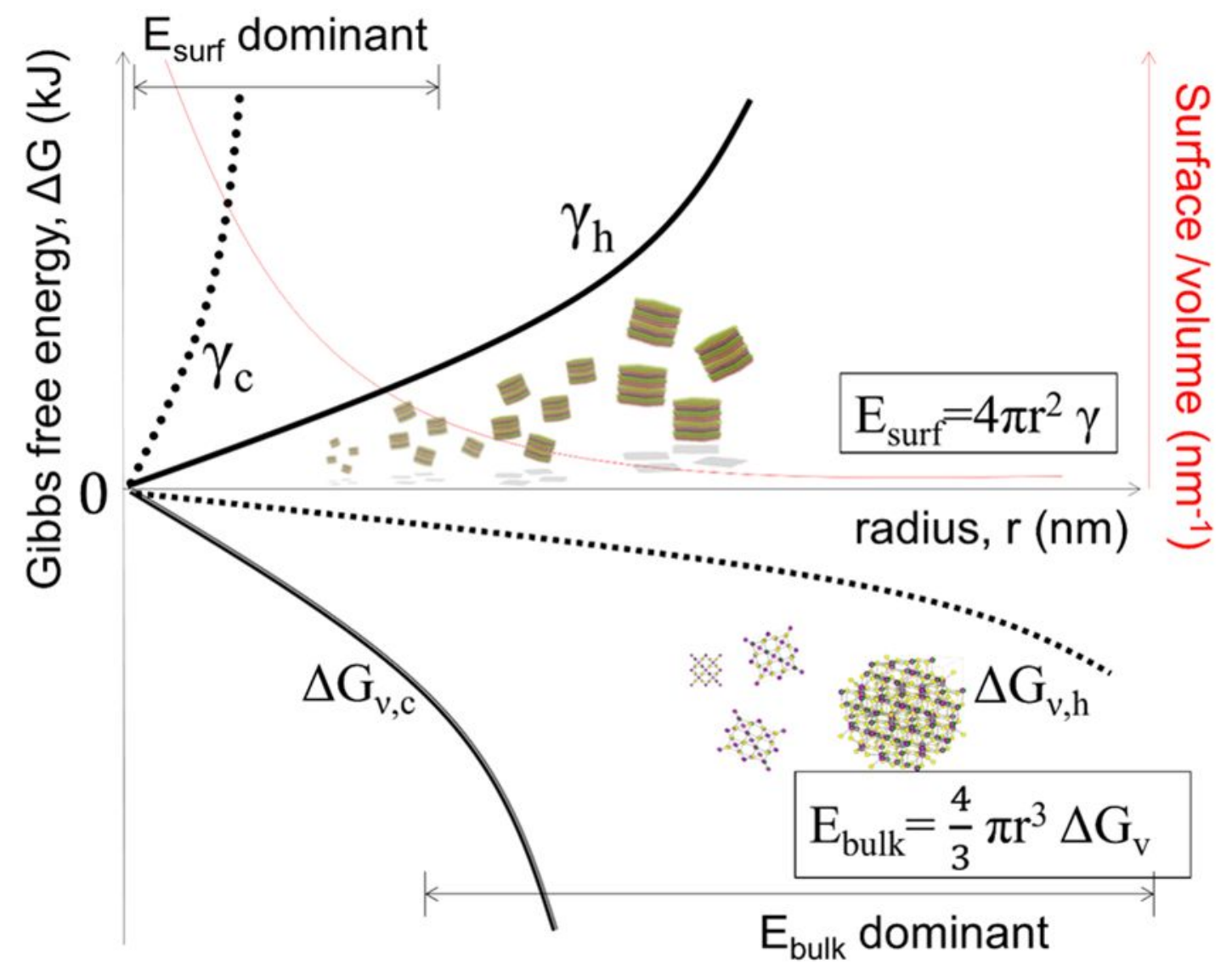

Figure S1. The mechanism of the formation of hexagonal and cubic (spinel) structure of $\mathrm{MIn}_{2} \mathrm{~S}_{4}$ is illustrated based on a previous study. ${ }^{1} \gamma_{c}$, and $\gamma_{h}$ respectively represent the surface tension of cubic (spinel) and hexagonal $\mathrm{MIn}_{2} \mathrm{~S}_{4}$, which is described with a surface energy equation. $\Delta \mathrm{G}_{\mathrm{v}, \mathrm{c}}$ and $\Delta G_{v, h}$ respectively refer to the Gibbs free energy per unit volume of cubic (spinel) and hexagonal $\mathrm{Mln}_{2} \mathrm{~S}_{4}$, which is described by a bulk energy equation. In the early stage of the nanocrystal growth, low surface tension drives the presence of the hexagonal phase, and, as growth process proceeds, a phase transition from hexagonal to cubic occurs to minimize the total energy of $\mathrm{Mln}_{2} \mathrm{~S}_{4}$. 


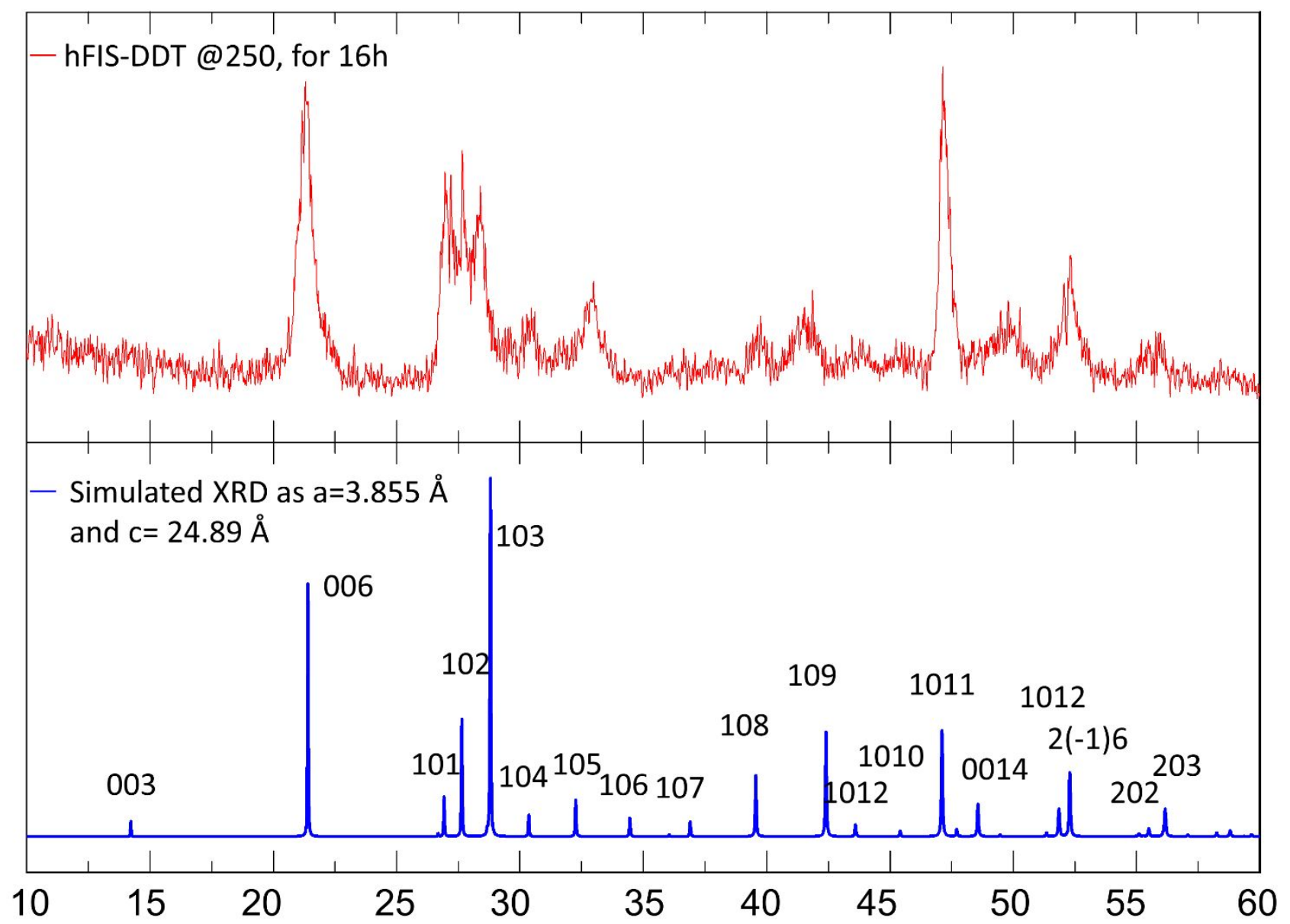

Figure S2. XRD result hFIS-DDT nanosheets synthesized at $250^{\circ} \mathrm{C}$ for 16 hours with a lateral size of around $200 \mathrm{~nm}$ (red line) was compared with simulated peaks referred to the lattice parameter value from ref. 38 (Space group: $P 6_{3} \mathrm{mc}$ (No.186), Unit cell parameter: $a=b=3.855 \AA, c=24.89 \AA$ ) 


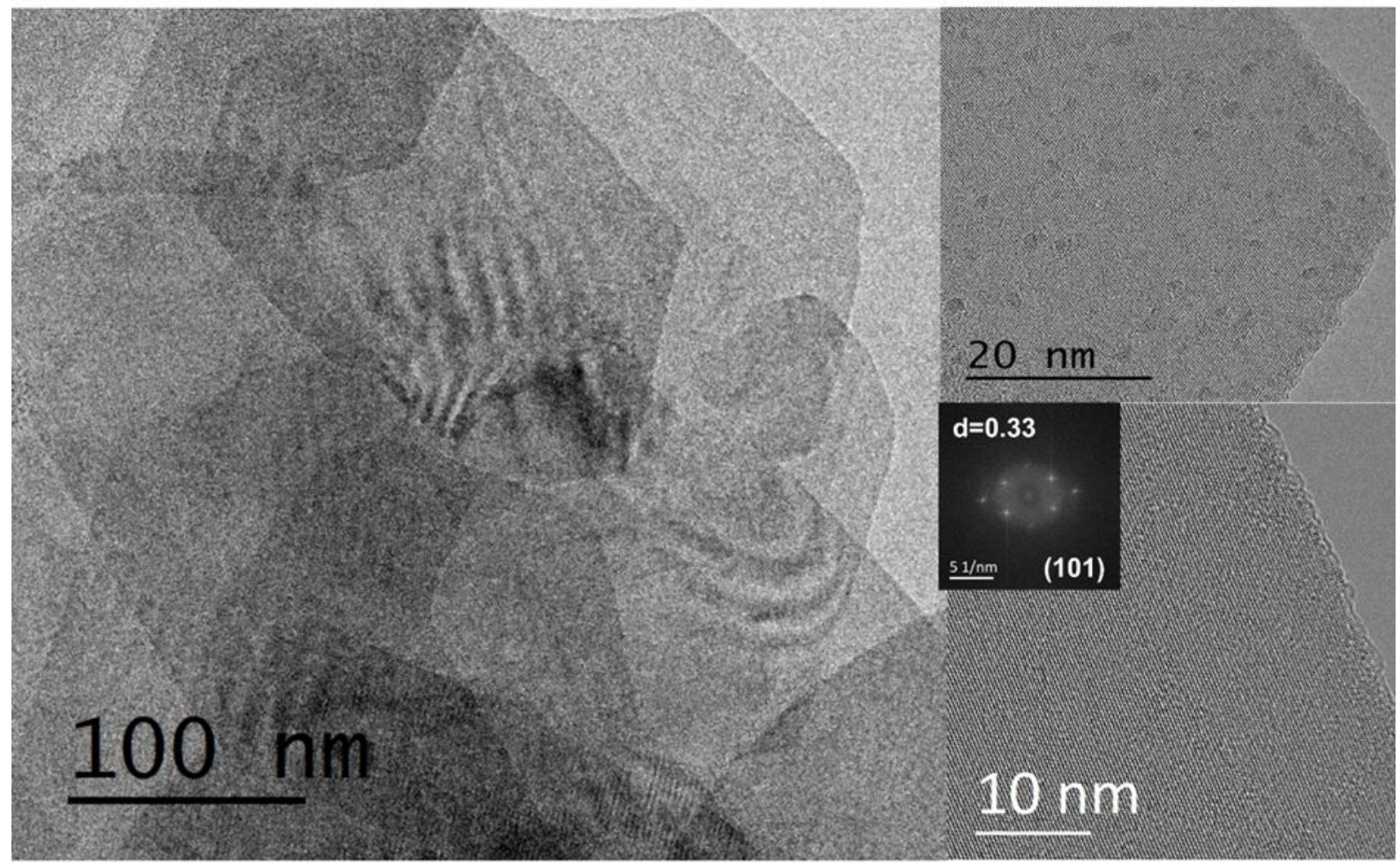

Figure S3. TEM images of hFIS-DDT nanosheets synthesized at $250^{\circ} \mathrm{C}$ for 16 hours with a lateral size of around $200 \mathrm{~nm}$. 


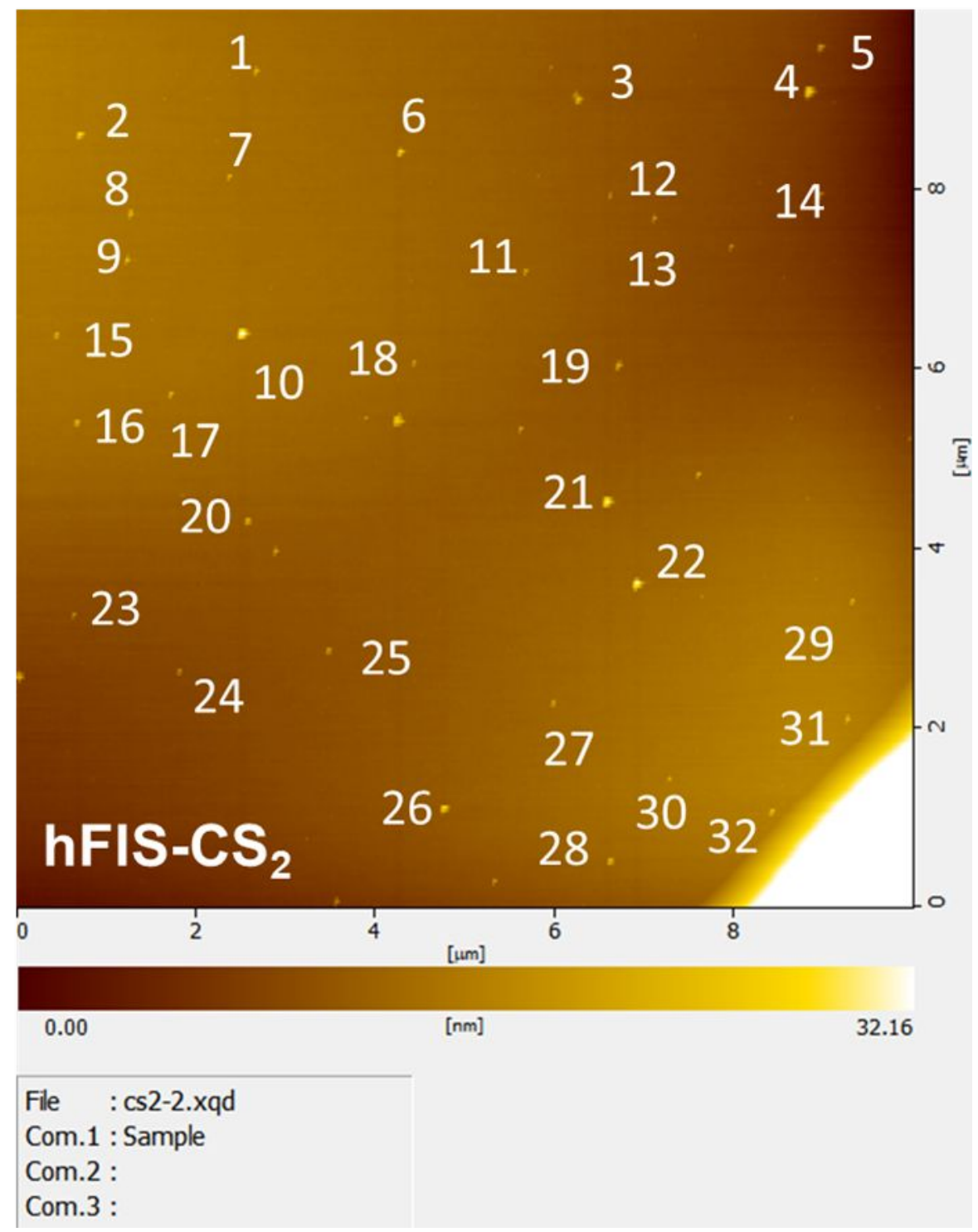

Figure S4. AFM images of hexagonal Feln $\mathrm{S}_{2} \mathrm{~S}_{4}$ nanosheets synthesized at $250{ }^{\circ} \mathrm{C}$ with $\mathrm{CS}_{2}$ for 10 hours for statistical analysis (Figure 4a, e) 


\begin{tabular}{|c|c|c|}
\hline & Height (nm) & Dimension (nm) \\
\hline 1 & 8.771 & 115.0031 \\
\hline 2 & 11.80088 & 134.6154 \\
\hline 3 & 11.56771 & 137.3626 \\
\hline 4 & 14.6398 & 179.4621 \\
\hline 5 & 10.71011 & 138.0495 \\
\hline 6 & 11.48 & 142.7045 \\
\hline 7 & 7.138087 & 58.22 \\
\hline 8 & 8.082445 & 80.89133 \\
\hline 9 & 8.618758 & 100.2653 \\
\hline 10 & 7.477440 & 161.1959 \\
\hline 11 & 7.313094 & 89.74359 \\
\hline 12 & 5.188238 & 80.89 \\
\hline 13 & 8.181986 & 101.1905 \\
\hline 14 & 7.471672 & 121.0341 \\
\hline 15 & 6.635681 & 115.4768 \\
\hline 16 & 9.56716 & 100.9615 \\
\hline 17 & 8.167073 & 107.5033 \\
\hline 18 & 12.50442 & 141.4835 \\
\hline 19 & 11.18747 & 118.6661 \\
\hline 20 & 10.27945 & 99.8932 \\
\hline 21 & 14.56639 & 138.1258 \\
\hline 22 & 18.28592 & 157.5092 \\
\hline 23 & 7.498673 & 103.0220 \\
\hline 24 & 7.576575 & 79.36508 \\
\hline 25 & 8.639154 & 94.32234 \\
\hline 26 & 13.95980 & 139.4231 \\
\hline 27 & 5.203418 & 103.0220 \\
\hline 28 & 9.096675 & 120.5739 \\
\hline 29 & 8.040875 & 80.58608 \\
\hline 30 & 4.20867 & 62.27106 \\
\hline 31 & 6.511293 & 77.83883 \\
\hline 32 & 5.162410 & 78.98352 \\
\hline
\end{tabular}

Table S1. The height and the dimension measurement result of Fel $\mathrm{n}_{2} \mathrm{~S}_{4}$ nanosheets synthesized at $250^{\circ} \mathrm{C}$ with $\mathrm{CS}_{2}$ for 10 hours for statistical analysis (Figure $4 \mathrm{a}, \mathrm{e}$ ) 


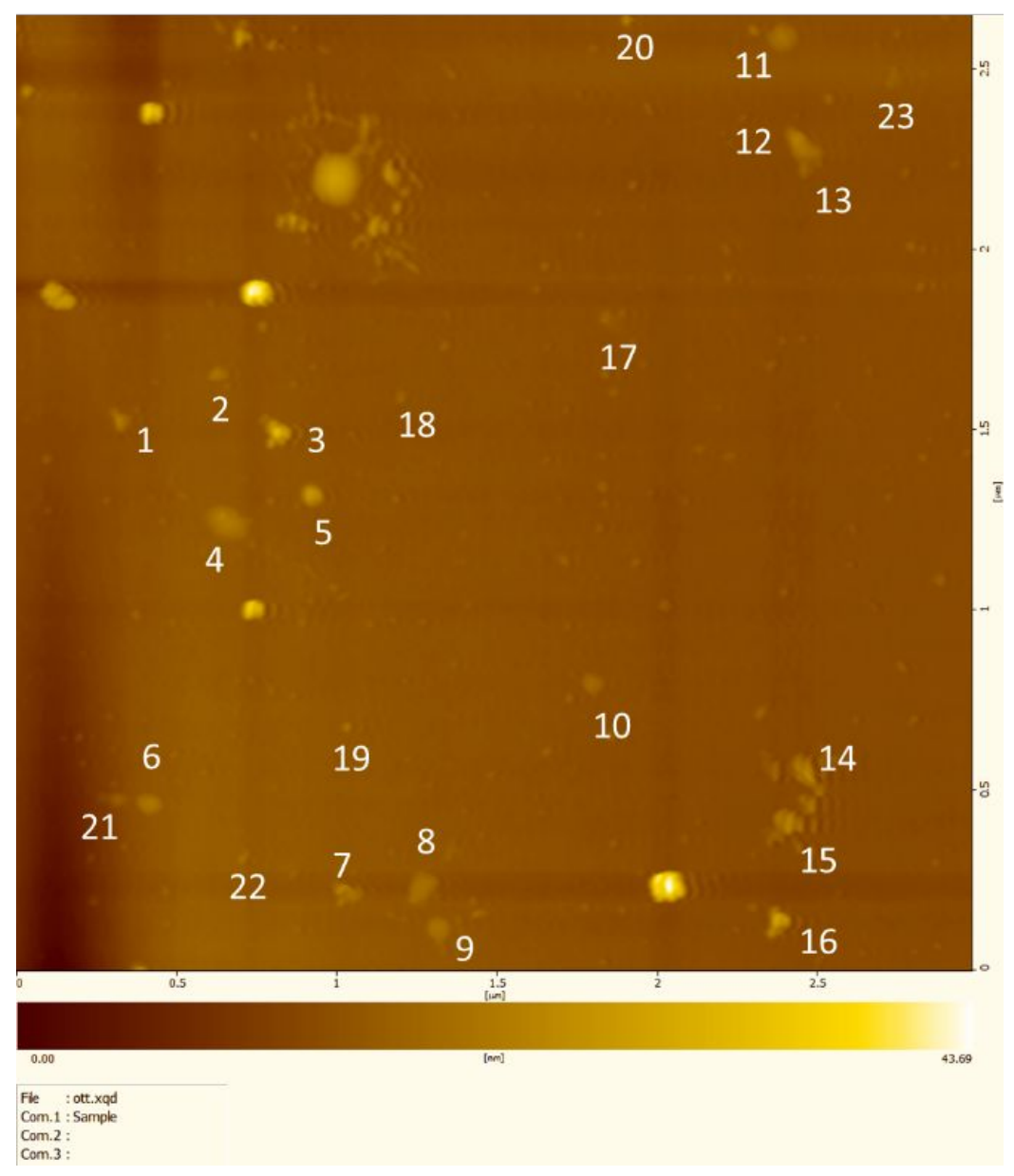

Figure S5. AFM images of hexagonal Feln $\mathrm{S}_{4}$ nanosheets synthesized at $250^{\circ} \mathrm{C}$ with OTT for 10 hours for statistical analysis (Figure $4 b, f$ ) 


\begin{tabular}{|l|c|c|}
\hline & Height (nm) & Dimension (nm) \\
\hline $\mathbf{1}$ & 7.33365 & 78.923 \\
\hline $\mathbf{2}$ & 4.52283 & 57.405 \\
\hline $\mathbf{3}$ & 13.3555 & 95.6807 \\
\hline $\mathbf{4}$ & 6.88713 & 111.737 \\
\hline $\mathbf{5}$ & 10.0274 & 95.464 \\
\hline $\mathbf{6}$ & 6.27241 & 93.6098 \\
\hline $\mathbf{7}$ & 4.48978 & 74.0057 \\
\hline $\mathbf{8}$ & 5.10518 & 125.203 \\
\hline $\mathbf{9}$ & 5.0967 & 105.852 \\
\hline $\mathbf{1 0}$ & 4.80831 & 78.2163 \\
\hline $\mathbf{1 1}$ & 5.51137 & 96.45 \\
\hline $\mathbf{1 2}$ & 6.93162 & 85.1672 \\
\hline $\mathbf{1 3}$ & 8.16707 & 107.503 \\
\hline $\mathbf{1 4}$ & 10.7737 & 100.939 \\
\hline $\mathbf{1 5}$ & 7.31347 & 89.8367 \\
\hline $\mathbf{1 6}$ & 9.16148 & 79.0607 \\
\hline $\mathbf{1 7}$ & 3.48115 & 102.593 \\
\hline $\mathbf{1 8}$ & 3.26387 & 63.5749 \\
\hline $\mathbf{1 9}$ & 5.27153 & 60.0278 \\
\hline $\mathbf{2 0}$ & 3.26387 & 63.5749 \\
\hline $\mathbf{2 1}$ & 5.27153 & 60.0278 \\
\hline $\mathbf{2 2}$ & 6.13021 & 52.9109 \\
\hline $\mathbf{2 3}$ & 2.83168 & 86.4411 \\
\hline
\end{tabular}

Table S2. The height and the dimension measurement result of Feln $\mathrm{S}_{4}$ nanosheets synthesized at $250^{\circ} \mathrm{C}$ with OTT for 10 hours for statistical analysis (Figure 4a, e) 


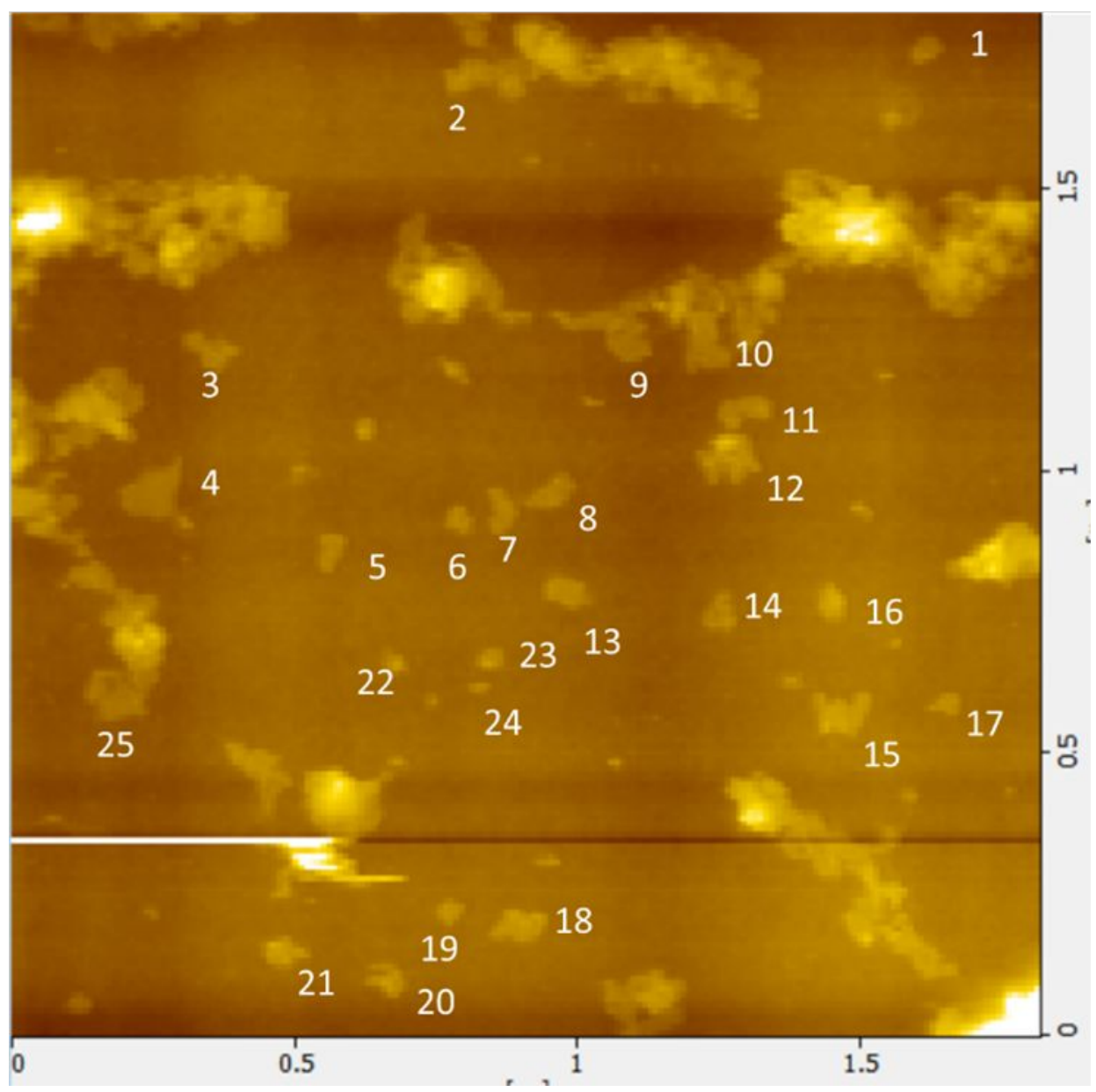

Figure S6. AFM images of hexagonal Feln $\mathrm{S}_{4}$ nanosheets synthesized at $250^{\circ} \mathrm{C}$ with DTT for 10 hours for statistical analysis (Figure 4c, g) 


\begin{tabular}{|c|c|c|}
\hline & Height (nm) & Dimension (nm) \\
\hline 1 & 2.383824 & 71.12332 \\
\hline 2 & 3.470974 & 78.60195 \\
\hline 3 & 2.679792 & 66.39194 \\
\hline 4 & 2.689469 & 123.6264 \\
\hline 5 & 2.816943 & 69.02473 \\
\hline 6 & 3.454714 & 75.89286 \\
\hline 7 & 2.930679 & 97.75641 \\
\hline 8 & 2.882081 & 92.03183 \\
\hline 9 & 2.518079 & 98.29060 \\
\hline 10 & 2.931723 & 68.45238 \\
\hline 11 & 3.688694 & 107.9823 \\
\hline 12 & 4.651461 & 131.4103 \\
\hline 13 & 2.790653 & 89.28571 \\
\hline 14 & 2.8966411 & 147.1222 \\
\hline 15 & 3.259123 & 113.0952 \\
\hline 16 & 3.604092 & 87.22527 \\
\hline 17 & 2.357556 & 39.7901 \\
\hline 18 & 3.534386 & 107.3718 \\
\hline 19 & 3.061747 & 67.30769 \\
\hline 20 & 3.966949 & 86.61477 \\
\hline 21 & 4.860190 & 98.63400 \\
\hline 22 & 2.423864 & 56.47131 \\
\hline 23 & 1.832619 & 73.71795 \\
\hline 24 & 1.443712 & 39.68254 \\
\hline 25 & 2.840571 & 126.6789 \\
\hline
\end{tabular}

Table S3. The height and the dimension measurement result of Feln $\mathrm{n}_{2} \mathrm{~S}_{4}$ nanosheets synthesized at $250^{\circ} \mathrm{C}$ 


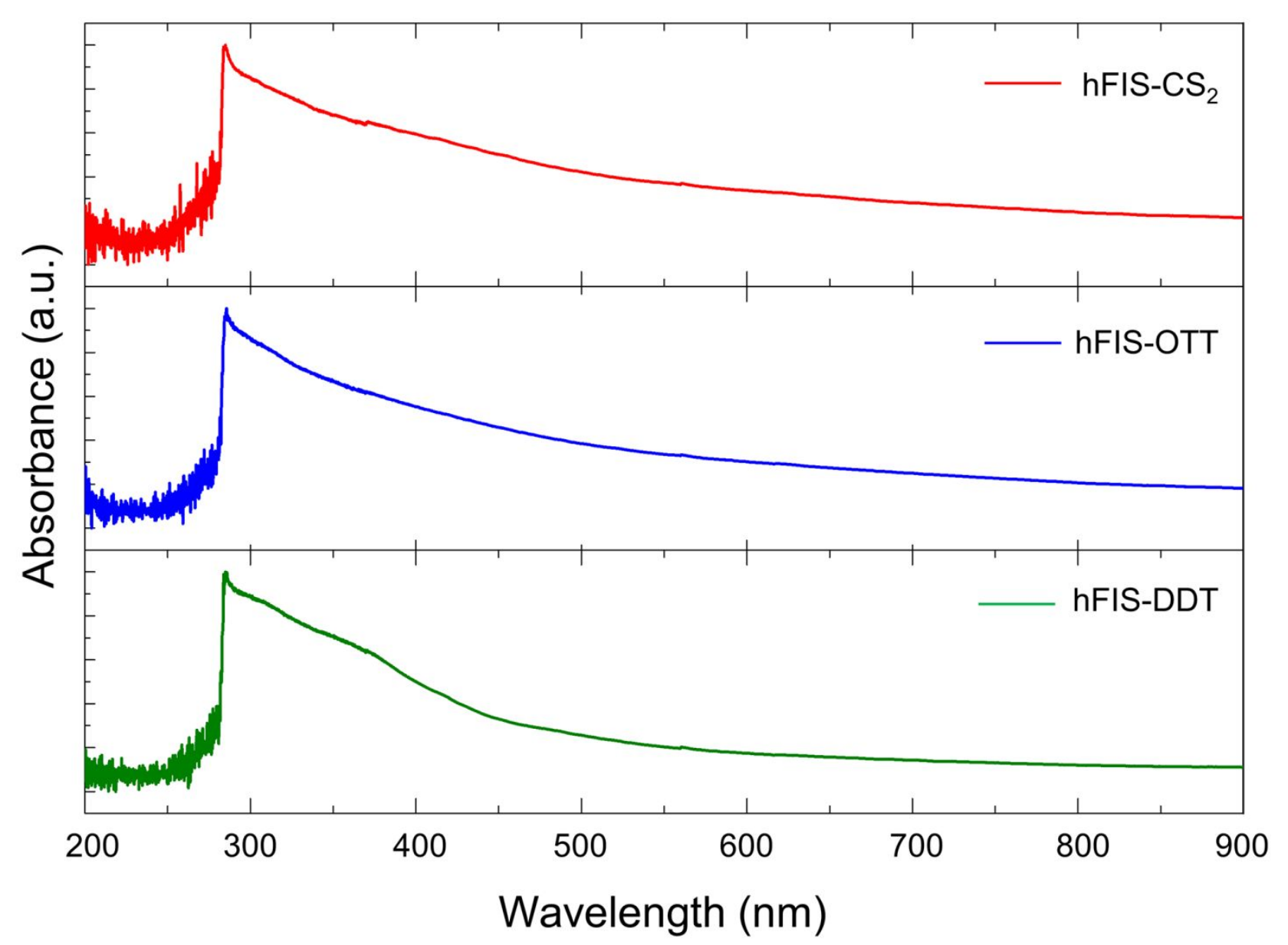

Figure S7. The absorption spectra of hFIS nanosheets synthesized with $\mathrm{CS}_{2}$ (red), OTT (blue), and DDT (green), respectively. 


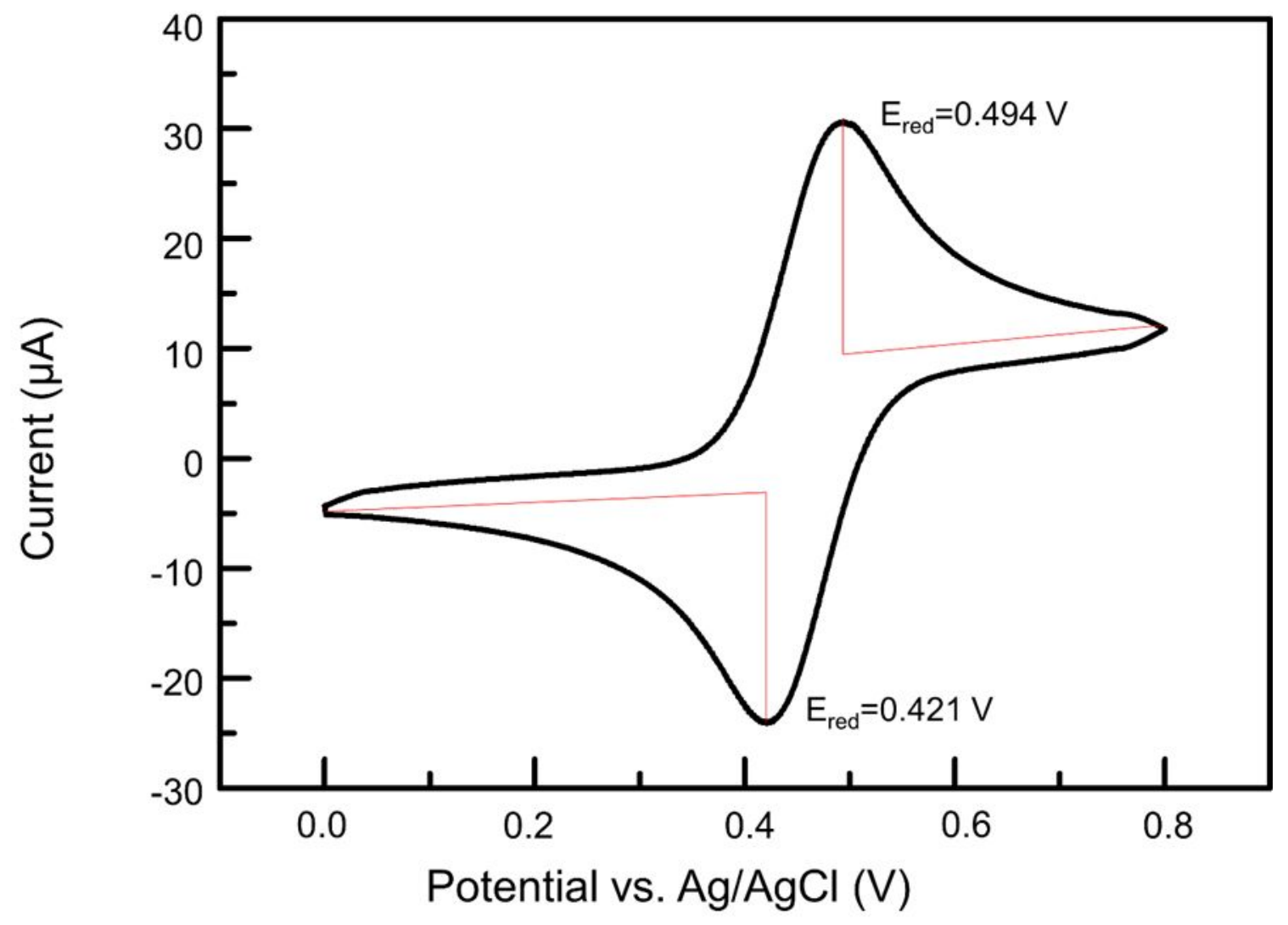

Figure S8. Redox potential of Ferrocene/Ferrocenium in a $0.1 \mathrm{M} \mathrm{TBAPF}$ acetonitrile solution showing a half wave potential at $0.4575 \mathrm{~V}\left(\mathrm{E}_{1 / 2}=1 / 2\left(\mathrm{E}_{\mathrm{red}}+\mathrm{E}_{\mathrm{ox}}\right)=(0.494+0.421)=0.4575 \mathrm{~V}\right)$. 
a
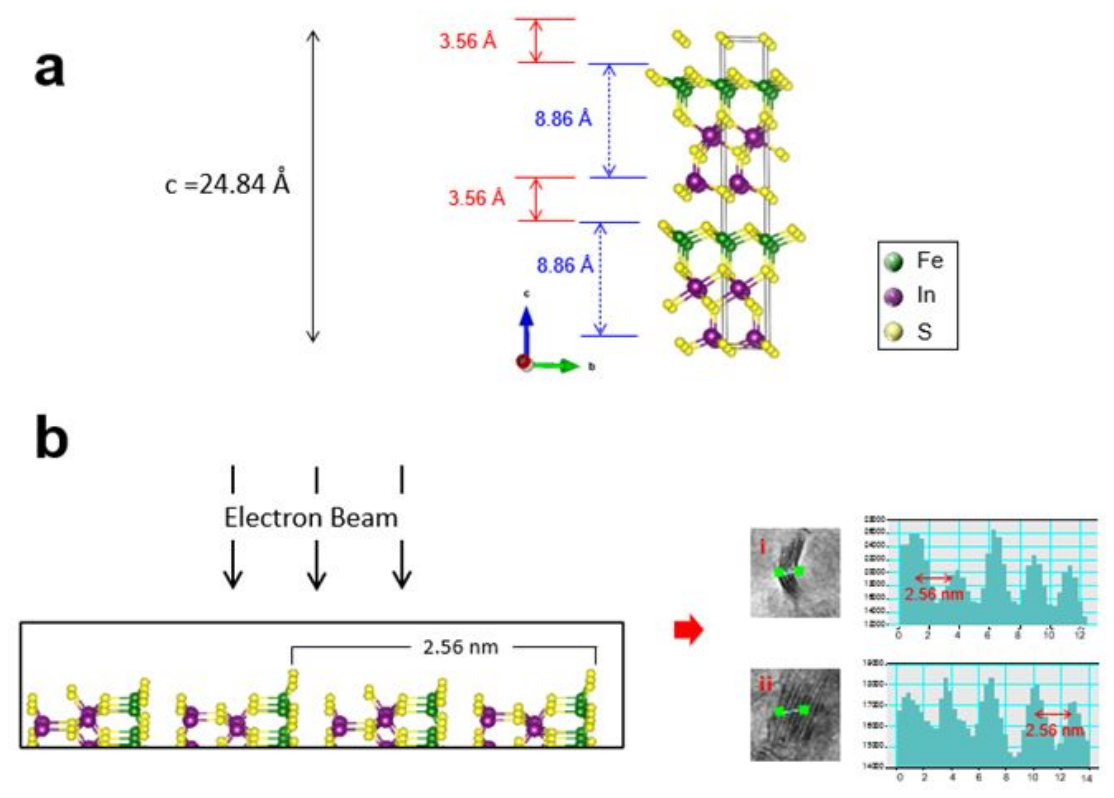

Figure S9. Hexagonal Feln ${ }_{2} \mathrm{~S}_{4}$ with $2 \mathrm{H}(\mathrm{ABAB}$...) stacking order with calculated the interlayer distance $3.56 \AA$. (a) The thickness of single layer hFIS after considering interlayer distance showed $8.86 \AA$. (b) Layered structure feature shown in TEM image in cross sectional view with $2.56 \mathrm{~nm}$ distance and expected the origin of the height profile.
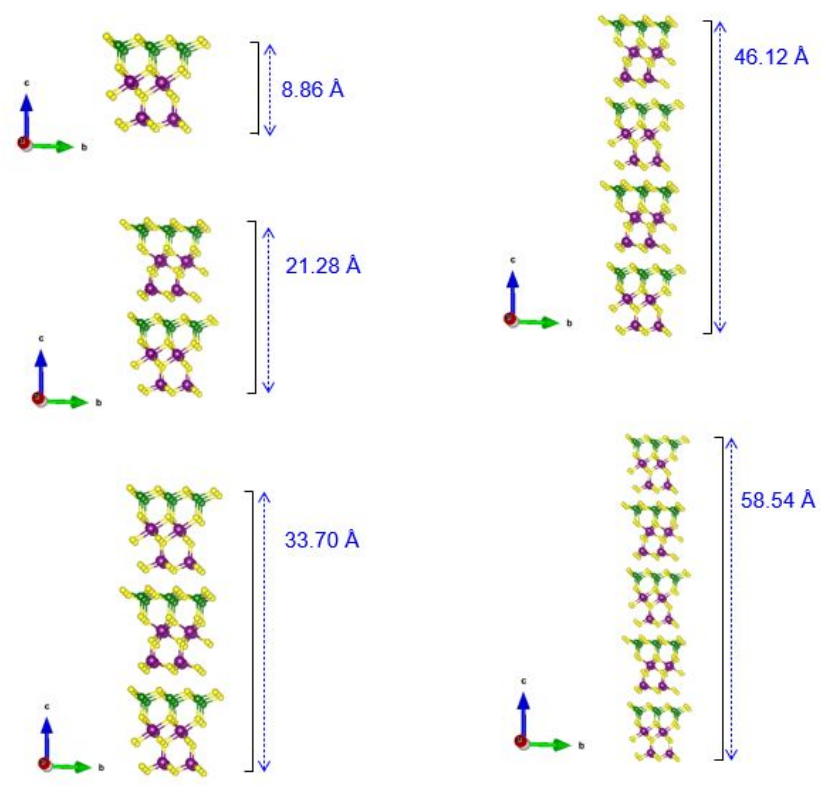

Figure S10. The thickness of hexagonal Feln ${ }_{2} \mathrm{~S}_{4}$ with various numbers of layers. 


\section{Unit cell parameter calculation:}

The lattice distance can be obtained by using Bragg's law: ${ }^{2}$

$$
2 \mathrm{~d} \sin \theta=\mathrm{n} \lambda
$$

Where $d$ is the lattice distance, $\theta$ is the corresponding diffracted angle, $\mathrm{n}$ is constant, and $\lambda$ is the wavelength of the diffracted $x$-ray. From the equation above, the lattice spacing values, $d$, were obtained as $d_{006}=0.414 \mathrm{~nm}$, and $d_{103}=0.332 \mathrm{~nm}$. To obtain the lattice constant for a hexagonal structure,

$$
\frac{1}{d^{2}}=\frac{4}{3}\left(\frac{h^{2}+h k+k^{2}}{a^{2}}\right)+\frac{l^{2}}{c^{2}}
$$

Where $\mathrm{h}, \mathrm{k}$, and $\mathrm{I}$ are expressed in the Miller index notation of the corresponding facet, and a and $\mathrm{c}$ are components for the primitive unit cell of the hexagonal structure. ${ }^{3}$

Unit cell parameter calculation result from XRD data (in Figure S2) of hFIS-DDT $250^{\circ} \mathrm{C}$ for $16 \mathrm{~h}$

\begin{tabular}{|l|l|l|l|l|l|l|}
\hline $\boldsymbol{h}$ & $\boldsymbol{k}$ & $\boldsymbol{I}$ & $\mathbf{2 \vartheta ( 9}$ & $\boldsymbol{d}(\mathbf{n m})$ & $\boldsymbol{c}(\mathbf{n m})$ & $\boldsymbol{a}(\mathbf{n m})$ \\
\hline $\mathbf{0}$ & $\mathbf{0}$ & $\mathbf{6}$ & 21.4 & 0.414883 & 2.489301 & \\
\hline $\mathbf{1}$ & $\mathbf{0}$ & $\mathbf{1}$ & 27.15 & 0.328181 & & 0.382288 \\
\hline $\mathbf{1}$ & $\mathbf{0}$ & $\mathbf{2}$ & 27.66 & 0.322245 & & 0.385231 \\
\hline $\mathbf{1}$ & $\mathbf{0}$ & $\mathbf{3}$ & 28.4 & 0.314014 & & 0.391726 \\
\hline $\mathbf{1}$ & $\mathbf{0}$ & $\mathbf{4}$ & 30.46 & 0.29323 & & 0.383878 \\
\hline $\mathbf{1}$ & $\mathbf{0}$ & $\mathbf{5}$ & 32.84 & 0.272503 & & 0.375979 \\
\hline $\mathbf{1}$ & $\mathbf{0}$ & $\mathbf{8}$ & 39.76 & 0.226525 & & 0.381527 \\
\hline $\mathbf{1}$ & $\mathbf{0}$ & $\mathbf{9}$ & 41.84 & 0.215732 & & 0.39805 \\
\hline
\end{tabular}

\section{Optical Band gap calculation: Tauc plot}

The optical band gap can be obtained by extrapolating slopes from the result of a conversion of the absorbance based on the equation below:

$$
[\alpha h v]^{p}=A\left(h v-E_{g}\right)
$$

Where $\alpha$ is the absorption coefficient, $h$ is Plank's constant, $v$ is the photon's frequency, $\mathrm{E}_{\mathrm{g}}$ is the band gap, $\mathrm{A}$ is a proportional constant, and $\mathrm{p}$ is a power index that is related to the optical absorption process; $p$ corresponds to allowed transitions, with a direct transition for $p=2$ and an indirect transition for $p=1 / 2 .{ }^{4,5}$ 
According to the SEPM theory, the relationship between the optical band gap and quasi-particle gap is described as:

$$
\varepsilon_{g a p}^{o p t}=\varepsilon_{g a p}^{q p}-J_{e 1, h 1}
$$

Where $\varepsilon_{g a p}^{o p t}$ is the optical band gap, $\varepsilon_{g a p}^{q p}$ is the quasi particle gap, and $J_{e 1, h 1}$ is the Coulombic interaction between the electron and hole. Since a polarization contribution $J_{e 1, h 1}^{p o l}$ is dependent on the dielectric constant of the environment, which is included in $J_{e 1, h 1}$, the optical band gap tends to depend on the dielectric environment during the operation. It is noticeable that the optical band is expected to be lower than the quasi particle gap due to $J_{e 1, h 1},{ }^{6}$ which is a critical clue to determine the type of band gap (direct or indirect allowed transition).

The HOMO and LUMO of hFIS calculated versus half wave potential of Ferrocene ( $\left.\mathrm{Fc} / \mathrm{Fc}^{+}\right)$is assumed at $-5.1 \mathrm{eV}$, which is close to the result from UPS measurement. ${ }^{7}$ Finally, both HOMO and LUMO position were determined as described as

$$
\begin{aligned}
\mathrm{HOMO}=\mathrm{I}_{p} & =-\left(\mathrm{E}_{\left[\text {onset, ox vs. } \mathrm{Fc}^{+} / \mathrm{Fc}\right]}+5.1\right)(\mathrm{eV}) \\
\mathrm{LUMO}=\mathrm{EA} & =-\left(\mathrm{E}_{\left[\text {onset, red vs. } \mathrm{Fc}^{+} / \mathrm{Fc}\right]}+5.1\right)(\mathrm{eV})
\end{aligned}
$$

\section{Characterization of Ultraviolet photoelectron spectroscopy (UPS) spectra}

The energy relation between the incident photon energy and electrons ejected to the vacuum can be described as

$$
\mathrm{E}_{k i n}=\mathrm{h} v-\mathrm{E}_{b}-\Phi_{s}
$$

Where $E_{k i n}$ is the kinetic energy of electrons, $h$ is Plank's constant, $v$ is the frequency, $E_{b}$ is the binding energy, and $\varphi_{s}$ is the work function of the sample. ${ }^{8}$ For a precise measurement of each energy state, all samples were characterized on an $\mathrm{Au}(\varphi=5.1 \mathrm{eV})$ deposited silicon wafer. 


\section{References}

1. Shi, Y.-F.; Wang, Y.; Wu, L.-M., Hexagonal MIn2S4 ( $M=M n, F e, C o):$ Formation and Phase Transition. J. Phys. Chem. C 2013, 117 (39), 20054-20059.

2. Bragg, W. H.; Bragg, W. L., The reflection of X-rays by crystals. Proceedings of the Royal Society of London. Series A, Containing Papers of a Mathematical and Physical Character 1913, 88 (605), 428438.

3. Kelly, A.; Knowles, K. M., Crystallography and crystal defects. John Wiley \& Sons: 2020.

4. Pankove, J. I., Optical processes in semiconductors. Courier Corporation: 1975.

5. Tauc, J., Optical properties and electronic structure of amorphous Ge and Si. Mater. Res. Bull. 1968, 3 (1), 37-46.

6. Inamdar, S. N.; Ingole, P. P.; Haram, S. K., Determination of Band Structure Parameters and the Quasi-Particle Gap of CdSe Quantum Dots by Cyclic Voltammetry. Chemphyschem 2008, 9 (17), 25742579.

7. Cardona, C. M.; Li, W.; Kaifer, A. E.; Stockdale, D.; Bazan, G. C., Electrochemical Considerations for Determining Absolute Frontier Orbital Energy Levels of Conjugated Polymers for Solar Cell Applications. Adv. Mater. 2011, 23 (20), 2367-2371.

8. Eland, J. H. D., Photoelectron spectroscopy: an introduction to ultraviolet photoelectron spectroscopy in the gas phase. Elsevier: 2013, 99-102. 